\title{
ATIVIDADE RESPIRATÓRIA DE SOLO TRATADO COM VINHAÇA E HERBICIDA (1)
}

\author{
ELI SIDNEY LOPES $\left({ }^{2}\right)$, SONIA CRISTINA PERON $\left({ }^{3}\right)$, \\ EDILBERTO PRINCI PORTUGAL ( ${ }^{3}$ ), OTÁVIO ANTONIO DE CAMARGO $\left({ }^{4}\right)$ \\ e SUELI DOS SANTOS FREITAS ${ }^{2}$ )
}

\begin{abstract}
RESUMO
Um experimento foi instalado a campo num Latossolo Vermelho-Escuro distrofico textura argilosa, da Estação Experimental de Limeira, em Cordeirópolis, SP, do Instituto Agronômico, para avaliar a atividade respiratória desse solo, quando enriquecido com vinhaça e tratado com o herbicida 2,4D. A vinhaça fora aplicada por quatro vezes, de seis em seis meses, em doses de 0,100 e $1.000 \mathrm{~m}^{3} / \mathrm{ha}$, sendo as determinaçōes feitas seis meses após a última aplicação. $O$ herbicida foi aplicado imediatamente antes do início das determinaçōes das atividades respiratórias e, o solo, mantido livre de vegetação. A atividade respiratória foi avaliada, medindo-se o $\mathrm{CO}_{2}$ coletado em solução de $\mathrm{KOH}$, em câmaras cilíndricas de PVC, em turnos de doze horas, durante quatro dias. As maiores taxas de $\mathrm{CO}_{2}\left(77,5 \mathrm{mg} \mathrm{CO} 2 / \mathrm{m}^{2} . \mathrm{h}\right)$ foram observadas no periodo diurno, e estiveram relacionadas com as temperaturas mais elevadas. Mesmo aos seis meses da aplicação da vinhaça, observou-se maior atividade respiratória nos tratamentos com o resíduo. $O$ herbicida $2,4 \mathrm{D}$, na dose de 3,0 litros /hectare, năo influenciou a atividade respiratoria.
\end{abstract}

Termos de indexação: vinhaça, herbicida 2,4D, respiração edáfica, microrganismos.

(1) Convênio IAC/MIC/STI/FUNAT. Recebido para publicaçāo em 16 de julho de 1985. (SP).

(2) Seção de Microbiologia do Solo, Instituto Agronômico (IAC), Caixa Postal 28, 13001 Campinas

(3) Biólogo estagiário, Seçāo de Microbiologia do Solo, IAC.

(4) Seção de Pedologia, IAC. Com bolsa de suplementação do CNPq. 


\section{INTRODUÇÃO}

A alternativa de aplicação de vinhaça ao solo, em vez de seu lançamento em rios, vem sendo recomendada como um meio de fertilização das culturas e constitui uma forma de eliminar uma das fontes poluidoras dos cursos de água. A incorporação de resíduos orgânicos ao solo promove aumentos na evolução do $\mathrm{CO}_{2}$, sendo parte desse aumento originária da decomposição da matéria orgânica do solo (ALEXANDER, 1961).

A vinhaça provoca alterações imediatas nas caracteristicas biológicas do solo. LIMA (1980) constatou aumentos na população microbiana, na taxa de decomposição da matéria orgânica, nitrificação, desnitrificação e fixação biológica do nitrogênio, em amostras de solo com adição de vinhaça e incubadas durante uma semana. Constatou, também, que a respiração, medida pela evolução de $\mathrm{CO}_{2}$, aumentou devido ao aumento da população microbiana do solo. Entretanto, efeitos do uso contínuo e/ou de longo prazo devidos à aplicação da vinhaça são desconhecidos.

A aplicação conjugada de vinhaça e herbicidas na lavoura canavieira tem constituído técnica recomendada, que vem sendo praticada por grande número de agricultores. Os herbicidas podem não afetar a atividade microbiana do solo, podem constituir uma fonte de carbono, ou ser tóxicos (ANDERSON, 1978).

O objetivo do presente trabalho foi verificar se a deposição de vinhaça no solo pode acarretar modificações duradouras na atividade microbiana do solo tratado ou não com herbicida.

\section{MATERIAL E MÉTODOS}

O experimento foi conduzido na Estação Experimental de Limeira em Cordeirópolis, SP, do Instituto Agronômico, em Latossolo Vermelho-Escuro distrófico textura argilosa, numa área experimental com três canteiros $(4 \times 4 \mathrm{~m})$ que receberam vinhaça nas seguintes dosagens: sem aplicação (0), aplicação de $100 \mathrm{~m}^{3} /$ ha/semestre (100), aplicação de $1.000 \mathrm{~m}^{3} / \mathrm{ha} /$ semestre $(1.000)$, durante 18 meses. A última aplicação fora efetuada em majo de 1981, seis meses antes da instalação deste experimento, e o solo vinha sendo mantido livre de vegetação através de cuidadosas capinas superficiais periódicas. $O$ quadro 1 apresenta os dados de algumas características químicas de amostras do solo das áreas experimentais correspondentes a cada dosagem.

Cada canteiro foi subdividido em seis partes, cujo centro foi utilizado para efetuar os tratamentos de aplicação de herbicida (sem ou com), em parcelas de $0,50 \times 0,50 \mathrm{~m}$. Cada tratamento foi, portanto, feito com três repetiçōes, de maneira inteiramente casualizada, dentro de cada canteiro. $O$ herbicida aplicado foi $o$ 2,4D (ácido 2,4 diclorofenoxiacético) em dosagem equivalente a 3,0 litros/hectare. 
A aplicação foi feita com solução aquosa, tendo sido distribuido, com regador, um litro por parcela. Nas parcelas correspondentes ao tratamento sem herbicida, adicionou-se apenas água. A atividade respiratória do solo foi avaliada pela medida do $\mathrm{CO}_{2}$ liberado durante quatro dias consecutivos, segundo GRISI (1978). Câmaras cilíndricas de PVC, com uma extremidade aberta, diâmetro de $30 \mathrm{~cm}$ e altura útil de $30 \mathrm{~cm}$, foram colocadas em cada parcela imediatamente após terem sido efetuados os tratamentos com o herbicida.

QUADRO 1. Características químicas das amostras de solo tratado com diferentes doses de vinhaça (RAIJ \& ZULLO, 1977)

\begin{tabular}{lccccccc}
\hline $\begin{array}{l}\text { Vinhaça } \\
\text { aplicada }\end{array}$ & $\mathrm{pH}$ & $\mathrm{C}$ & $\mathrm{Al}^{3+}$ & $\mathrm{Ca}^{2+}$ & $\mathrm{Mg}^{2+}$ & $\mathrm{K}^{+}$ & $\mathrm{P}$ \\
\hline $\mathrm{m}^{3} / \mathrm{ha}$ & & $\%$ & \multicolumn{2}{c}{$\mathrm{meq} / 100 \mathrm{~cm}^{3}$} & $\mu \mathrm{g} / \mathrm{ml}$ & TFSA \\
$0 \ldots$ & 4,5 & 1,6 & 1,5 & 0,3 & 0,1 & 22 & 1,5 \\
$100 \ldots$ & 4,4 & 1,7 & 1,7 & 0,5 & 0,2 & 117 & 0,0 \\
$1.000 \ldots$ & 5,2 & 2,1 & 0,6 & 1,5 & 0,8 & 312 & 0,0 \\
\hline
\end{tabular}

$\mathrm{O} \mathrm{CO}_{2}$ desprendido foi coletado em solução de $\mathrm{KOH} 0,5 \mathrm{~N}$. Esta solução era trocada às $7 \mathrm{e}$ às 17 horas, para coleta do $\mathrm{CO}_{2}$ a ser liberado no período diurno e noturno respectivamente. A solução de $\mathrm{KOH}$ contendo o $\mathrm{CO}_{2}$ evoluído foi armazenada em recipientes hermeticamente fechados e $\mathrm{O} \mathrm{CO}_{2}$ foi posteriormente determinado por titulometria, segundo metodologia utilizada por GRISI (1978).

\section{RESULTADOS E DISCUSSÃO}

A umidade do solo permaneceu favorável à atividade microbiana, devido à ocorrência de chuvas antes e durante a instalação do ensaio.

$\mathrm{Na}$ figura 1, observa-se que as taxas de evolução de $\mathrm{CO}_{2}$ foram maiores nos períodos diurnos (média de $77,5 \mathrm{mg} \mathrm{CO} 2 / \mathrm{m}^{2}$.h) do que nos noturnos (média de $58,8 \mathrm{mg} \mathrm{CO} 2 / \mathrm{m}^{2}$.h), parecendo haver relação com a variação de temperatura superficial do solo, que favoreceria a atividade microbiana como um todo. Convém observar que este experimento foi instalado em área mantida livre de vegetação, para evitar a liberação de $\mathrm{CO}_{2}$ pelas raízes que interferiria na análise. Em presença de vegetação, verificam-se normalmente maiores taxas noturnas de evolução de $\mathrm{CO}_{2}$ (MEDINA, 1969). 


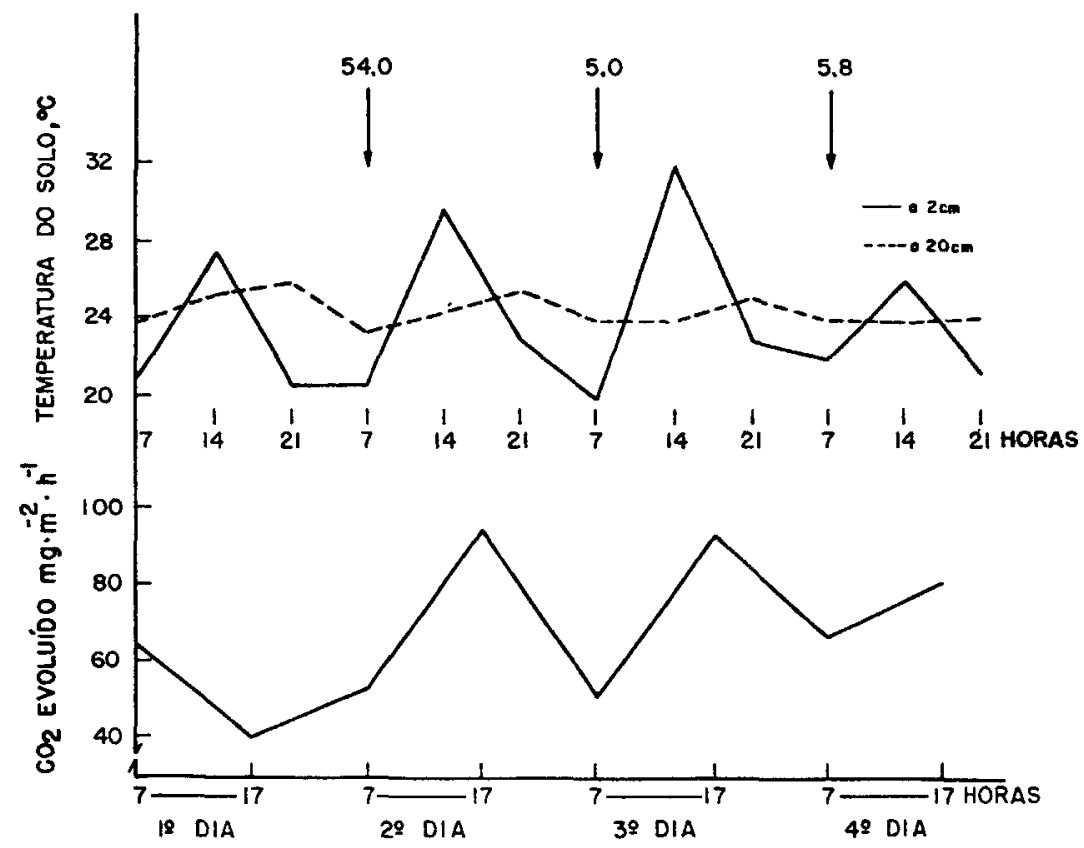

FIGURA 1. Variação diária de $\mathrm{CO}_{2}$ desprendido durante o período experimental de quatro dias (média de três repetições). Os números acima das setas da parte superior referem-se à precipitação pluvial $(\mathrm{mm})$ do dia marcado pela seta.

$\mathrm{Na}$ figura 2 encontram-se os valores da taxa de evolução do $\mathrm{CO}_{2}$, no perodo experimental, dos canteiros com as diferentes doses de vinhaça, para as áreas com e sem aplicação do herbicida. $\mathrm{A}$ evolução de $\mathrm{CO}_{2}$ aumentou significativamente com as doses de vinhaça (Figuras 2 e 3). Esse efeito da vinhaça, portanto, foi observado mesmo depois de seis meses da sua aplicação ao solo, quando a avaliação foi feita. Não se constataram diferenças estatisticamente significativas entre os tratamentos com e sem herbicida. Pode-se concluir que o 2,4D não teve efeito sobre a atividade microbiana do solo, na quantidade em que foi aplicado e na presença ou na ausência de vinhaça.

A evolução de $\mathrm{CO}_{2}$, conforme indicado na figura 3, aumentou após o primeiro dia para todos os tratamentos, sendo sempre maior para as maiores doses de vinhaça. Considerando-se que a aplicação de vinhaça fora feita seis meses antes, não se explica o aumento inicial da respiração edáfica, já que no primeiro dia não ocorreu qualquer manuseio que pudesse ter induzido um aumento na atividade microbịana, seguido de estabilização, em tão curto período. Deve-se considerar, no entanto, que o experimento foi de curta duração, não permitindo conclusões definitivas. 


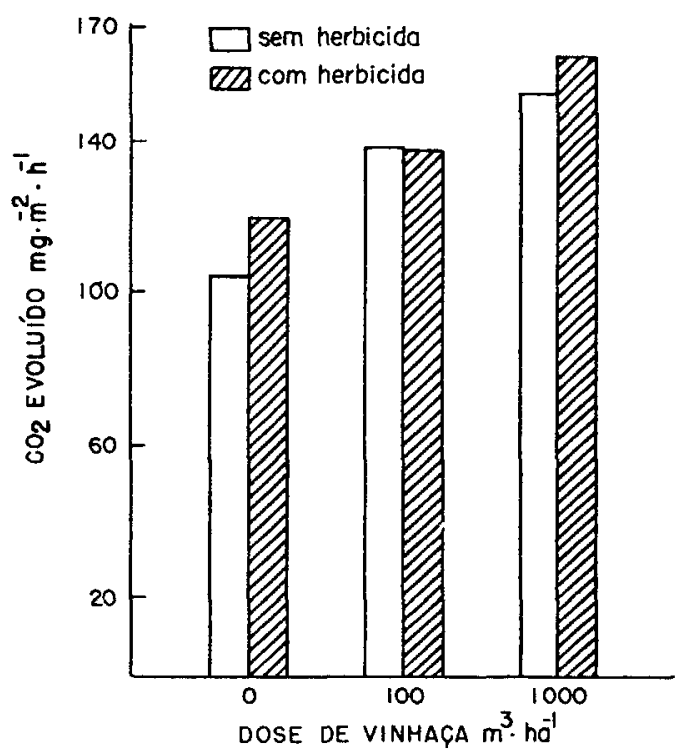

FIGURA 2. Evolução de $\mathrm{CO}_{2}$ no período de quatro dias, nos canteiros com as diferentes doses de vinhaça, com e sem aplicação do herbicida.

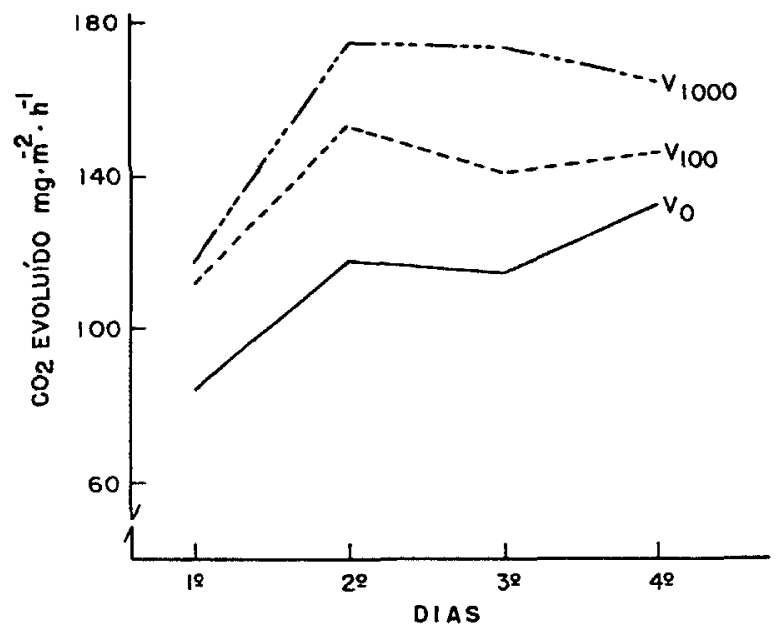

FIGURA 3. Evolução de $\mathrm{CO}_{2}$ no período experimental, nos canteiros com as diferentes doses de vinhaça aplicada 6 meses antes: $0=$ sem vinhaça; $100=100 \mathrm{~m}^{3} /$ ha; $1.000=$ $1.000 \mathrm{~m}^{3} / \mathrm{ha}$. 


\section{CONCLUSŌES}

Seis meses após a sua aplicação, a vinhaça ainda influenciou a atividade respiratória do solo, aumentando-a, quando nas dosagens de 100 e $1.000 \mathrm{~m}^{3} / \mathrm{ha}$.

O herbicida 2,4D, na dosagem de 3 litros/ha, não influenciou a atividade respiratória do solo.

\section{SUMMARY \\ RESPIRATORY ACTIVITY OF STILLAGE AND HERBICIDE TREATED SOIL}

A field experiment was carried out on a clayey dystrophic Dark-Red Latosol at the Limeira Experimental Station of the Instituto Agronômico (Cordeirópolis, State of São Paulo, Brazil), to evaluate the respiratory activity of the soil when enriched with stillage and treated with the herbicide $2,4 \mathrm{D}$. Stillage was applied three times with a sixmonth interval at 0,100 , and $1,000 \mathrm{~m}^{3} / \mathrm{ha}$, and the $\mathrm{CO}_{2}$ evolution measurements began six months after the last application. The soil was maintained free from vegetation all over the experiment. The $\mathrm{CO}_{2}$ was trapped by $\mathrm{KOH}$ in PVC cilindric chambers, and collected every 12 hours for analysis, during four days. Highest $\mathrm{CO}_{2}$ evolution rates (77.5mg $\mathrm{CO}_{2} / \mathrm{m}^{2} . \mathrm{h}$ ) were observed in daytime. Even six months after stillage application, the rate of $\mathrm{CO}_{2}$ evolution was higher in treated soils. The herbicide, applied at 3,0 $\mathrm{l} / \mathrm{ha}$, showed no significant influence on soil respiratory activity.

Index terms: stillage, herbicide $2,4 \mathrm{D}$, soil respiration, microorganisms.

\section{REFERÊNCIAS BIBLIOGRÁFICAS}

ALEXANDER, M. Introduction to soil Microbiology. New York, John Wiley, 1961. 472p.

ANDERSON, J.R. Pesticide effects on non-target microorganisms. In: HILL, I.R. \& WRIGHT, S.J.L. Pesticide Microbiology. London, Academic Press, 1978. p.313$-533$.

GRISI, B.M. Método químico de medição da respiração edáfica: alguns aspectos técnicos. Ciência e Cultura, São Paulo, 30:82-88, 1978.

LIMA, I.T. Efeitos da aplicação da vinhaça sobre a microflora do solo. Rio de Janeiro, UFRRJ, 1980. 150p. Tese (Mestrado)

MEDINA, E. Respiración edáfica de algunas comunidades tropicales. Boletín de la Sociedad Venezuelana de Ciencias Naturales, Caracas, 28:211-230, 1969.

RAIJ, B. van \& ZULLO, M.A.T. Métodos de análise de solo para fins de fertilidade. Campinas, Instituto Agronômico, 1977. 16p. (Circular, 63) 